\title{
Rapid Extraction of Disaster-Affected Areas Based on Affected Objects Classification System for Disaster Mitigation
}

\author{
Wen Dong \\ State Key Laboratory of Remote Sensing Science \\ Institute of Remote Sensing and Digital Earth, CAS \\ Beijing, China \\ e-mail: dongwen01@radi.ac.cn
}

\author{
Haiping Yang \\ State Key Laboratory of Remote Sensing Science \\ Institute of Remote Sensing and Digital Earth, CAS \\ Beijing, China \\ e-mail: yanghp@radi.ac.cn
}

\begin{abstract}
Remote sensing images have been a main information source of the pre-disaster monitoring, disaster prediction and rapid assessments of disasters. However, the existing affected objects classification system, which is designed for the post-disaster survey, hardly meets the requirements of applications in disaster mitigation. In this paper, we proposed the construction scheme of affected objects classification system for disaster mitigation, considering of characteristics of data disasters and affected objects. In the framework of this classification scheme, we specified the nomenclature for floods, which is then used in the rapid extraction of the flooded area in Yuyao. And the flooded area is extracted through change detection. Experiments showed that the classification system can be applied in classifying the affected bbjects directly, which is the basis of rapid assessment with remote sensing images.
\end{abstract}

Keywords-remote sensing information extraction; disaster mitigation; affected bbjects; classification system; flood;

\section{INTRODUCTION}

Natural disasters occurred frequently all around China, which caused serious damage to China's economic construction and social development. For example, in 2014, natural disasters caused 24.35 million people affected, 1583 people killed and 235 people missing; they also resulted in 337.38 billion yuan direct economic loss [1]. Even the direct economic loss hit 580.84 billion yuan in 2013, which is the largest amount since 2000 [2].

In recent years, in addition to traditional field surveys and statistical methods, some new methods and technologies are gradually applied in disaster mitigation. Remote sensing technology, as an example, has been studied for a long time in disaster monitoring and prediction of large areas and has been applied in disaster reduction business, because of its real-time, wide coverage, low cost, etc.[3-5]. As the temporal and spatial resolution of remote sensing images are improving, they are becoming the main data sources for disaster monitoring and forecasting. However, the current disaster evaluation system, which is mainly supported by survey data after disaster, can hardly provide disaster based solutions with remote sensing. In this paper, the construction scheme of affected objects classification system for disaster mitigation is constructed according to the characteristics of disasters and their visual representation in multi-source remote sensing data. And then this classification system is used in automatic classification of remote sensing images covered disaster areas, which is the basis of rapidly defining disaster-affected areas. Experiments are performed on HJ-1 images before and after floods in Yuyao. Then the accuracy and efficiency are evaluated.

\section{CONSTRUCTION OF AFFECTED OBJECTS \\ CLASSIFICATION SYSTEM FOR DISASTER MITIGATION}

\section{A. Classification Methods and Classification System}

At present, the application of remote sensing for disaster reduction is mainly focused on the extraction methods of different affected objects [6-9]. However, the affected objects classification system for disaster mitigation with remote sensing images and the role of remote sensing in disaster reduction process still need to be studied further. In this paper, a faceted classification is used to classify the affected objects that cannot be accurately expressed by a single classification criteria. And the facets include types of natural disasters, hazard bearing bodies and damage states.

The main affected objects differs in various types of natural disasters. From the practical point of view, the natural disaster classification scheme in China is chosen here. And we mainly focus on meteorological, hydrological and geological disasters. Specifically, as shown in Fig.1, 6 kinds of natural disasters, including earthquake, floods, typhoons, drought, landslide and low temperature disaster, are considered.

Natural disasters precipitate damage to property, injuries, or both. At present, disaster statistics contain casualties, housing damage, loss of personal property and national economic loss. The amount of casualties and economic loss cannot be obtained directly through remote sensing images due to the limitation of the remote sensing technology. They are mainly estimated through indirect calculations. In this paper, we mainly focus on the hazard bearing bodies that can be seen on the remote sensing images. Taking into account the needs of post-disaster damage assessment, hazard bearing bodies are divided into four categories including infrastructure, agriculture 
facilities, buildings and natural resources and environment (Fig .1).

Currently, most of the qualitative and quantitative damage assessments are based on the calculation from sampling survey data or post disaster statistical data [1011]. Ideally, post-disaster information extracted from remote sensing images can provide spatially and temporally better sources for damage assessments than survey data and statistical data do. However, the current image interpretation can not meet the needs of damage assessments, which is mainly evaluated qualitatively. In this paper, to meet the needs of damage assessments, the damage states of the affected objects are divided into four levels, good, low damage, serious damage and complete damage, by considering the existing disaster statistics and other related standards.

Considering the actual observation capability of remote sensing images and application requirements of disaster reduction, we selected the affected objects which can be identified by visual interpretation or automatic identification methods. Then the faceted classification scheme is used to build the affected objects classification system for disaster mitigation. .

Fig .1 shows the classification scheme for disaster mitigation with remote sensing images. We can see that the scheme contains three aspects, including natural disaster types, hazard bearing bodies and damage states Natural disaster types can be added flexibly according to the actual types of disasters. And the subcategories of hazard bearing bodies and damage states can be further detailed with the improving remote sensing observation technology. Take the types of buildings as an example, from the 10-meter images, built-up areas comprising of buildings are observed; from the 1-meter images, one single building can be seen.

\section{B. Affected Objects Classification System for Floods}

For any kind of natural disasters, its corresponding affected objects classification system can be detailed or simplified according to the classification system shown in Fig.1.

In this section, affected objects classification system for floods is further detailed. From the aspect of hazard bearing bodies, floods have a strong impact on agricultural lands, which results in crop damage. Particularly, we focus on the cultivated land, orchard and greenhouses. In terms of natural resources and environment, only land resources, including grasslands and forests, and water resources, including rivers and lakes, are detailed. With respect to the buildings and infrastructures, they are divided according to the spatial resolution of remote sensing images. From the aspect of damage states, taking into account of both the characteristics of floods and the levels of damage states with remote sensing technology, the four categories are merged into two categories, intact and damaged.

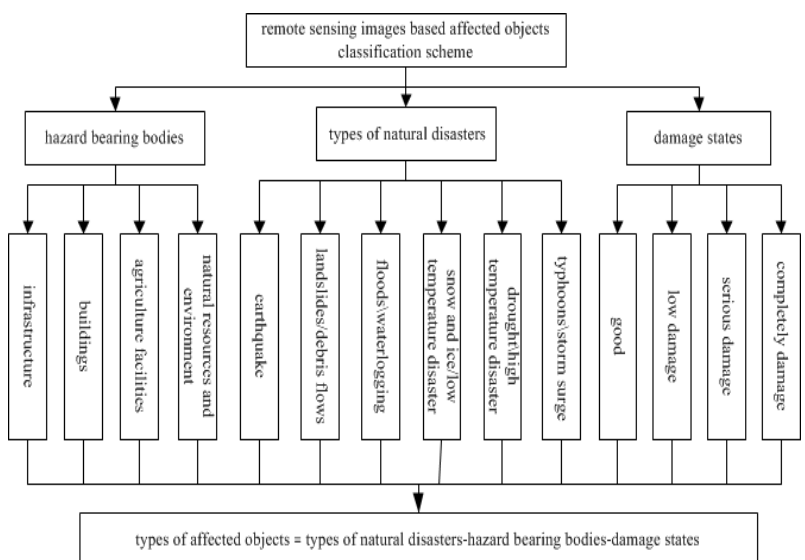

Figure 1. Affected objects classification system for disaster mitigation with remote sensing images

After floods, the types of affected objects in flooded areas can be determined according to the spatial resolution of remote sensing data based on the affected objects classification system for floods, which is used to support the classification of affected objects. Then the flooded areas are extracted through change detection.

\section{RAPID EXTRACTION OF THE FLOODED AREA}

\section{A. Overview of the flooded area}

Yuyao City in Zhejiang suffered the most serious floods since the founding of New China on October 7, 2013 because of the influence of the strong typhoon No. 23, "Buffett". 21 towns and streets are affected and 145 villages and communities were surrounded in Yuyao. 832,870 people were affected, 25,650 houses were damaged seriously and 61,665 people were transferred to safe places. Statistics shows the direct economic loss of Yuyao hit 6.991 billion Yuan.

HJ-1 satellite images on September 10, 2013 (Fig. 2) and October 12, 2013 (Fig. 3) are used in the experiment. The image spatial resolution is 30 meters.

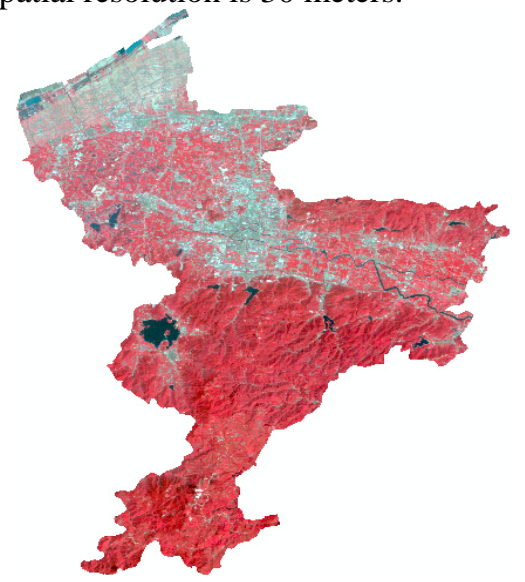

Figure 2. HJ-1 images covered Yuyao before floods 


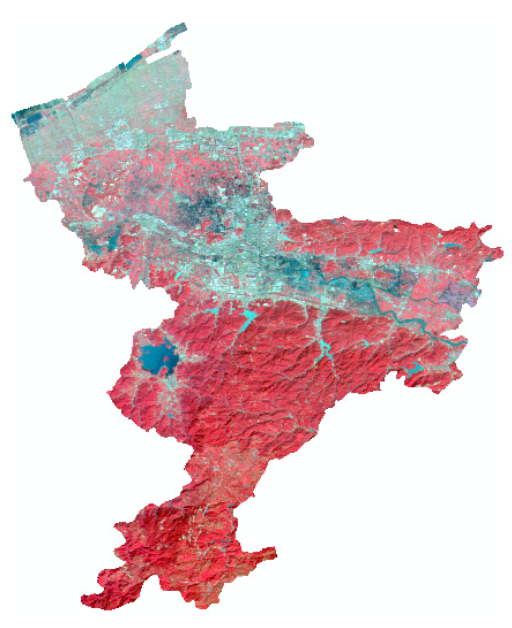

Figure 3. HJ-1 images covered Yuyao after floods

\section{B. Extraction of Flooded Area}

The process of the affected areas extraction by floods in Yuyao is shown in Fig. 4. We determine the affected objects based on the affected objects classification system for floods considering $\mathrm{HJ}-1$ satellite image spatial resolution, spectral information and other characteristics for this application in Yuyao floods. Then automatic classification is carried out on pre-disaster and postdisaster images under the support of the sample library. Finally, changed detection is used to extract the flooded areas.

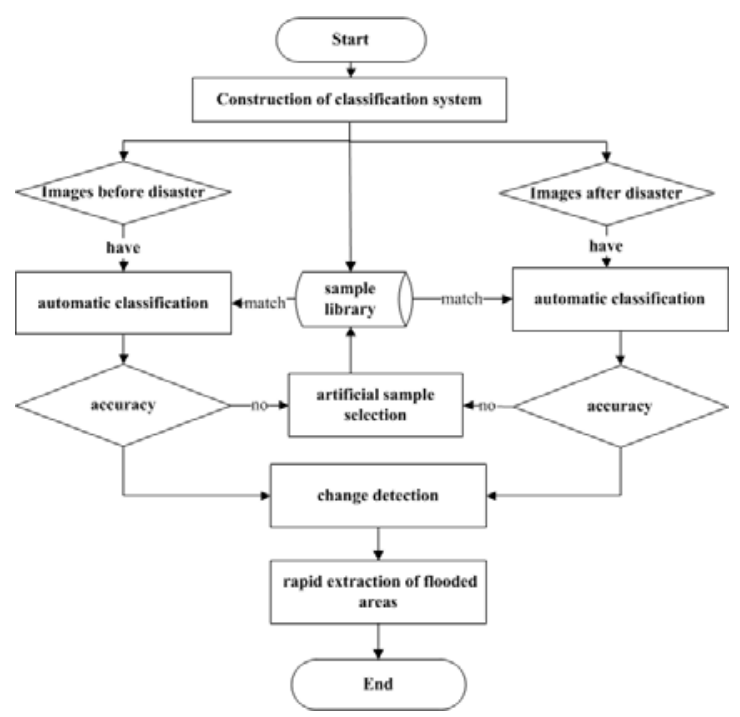

Figure 4. Process of the affected areas extraction by floods in Yuyao

The spatial resolution of $\mathrm{HJ}-1$ remote sensing data is 30 meters, therefore it is hardly to identify a single building or other fine categories with these images. After this fact is considered, the affected objects nomenclature for image classification is listed in Table 1. There are two levels in this classification system. In Level 1, there are four types including vegetation, water, artificial surfaces and unused lands. In Level 2, types of vegetation and artificial surfaces are detailed based on the damage states.
TABLE 1 AUTOMATIC CLASSIFICATION SYSTEM AND ITS CODING

\begin{tabular}{|l|l|}
\hline \multicolumn{1}{|c|}{ Level 1 } & \multicolumn{1}{c|}{ Level 2 } \\
\hline \multirow{2}{*}{1 vegetation } & 11 intact \\
\cline { 2 - 2 } & 12 damaged \\
\hline 2 water & 21 water \\
\hline \multirow{2}{*}{5 artificial surfaces } & 51 intact \\
\cline { 2 - 2 } & 52 damaged \\
\hline 6 unused lands & 61 bare \\
\hline
\end{tabular}

According to Table 1, we carry out the classification of the images before and after disaster with the support of sample database. The classification results are shown in Fig.5 and Fig.6.

Farmland, buildings and infrastructures are flooded after the floods. Therefore, vegetation, buildings and other objects mainly change from intact type into damaged type or water type in image data. On the basis of this, we conclude that the main categories changes of flooded areas include (1) intact vegetation to damaged vegetation, (2) intact artificial surfaces to damaged artificial surfaces, (3) intact vegetation to water, (4) intact artificial surfaces to water and (5) bare lands to water.

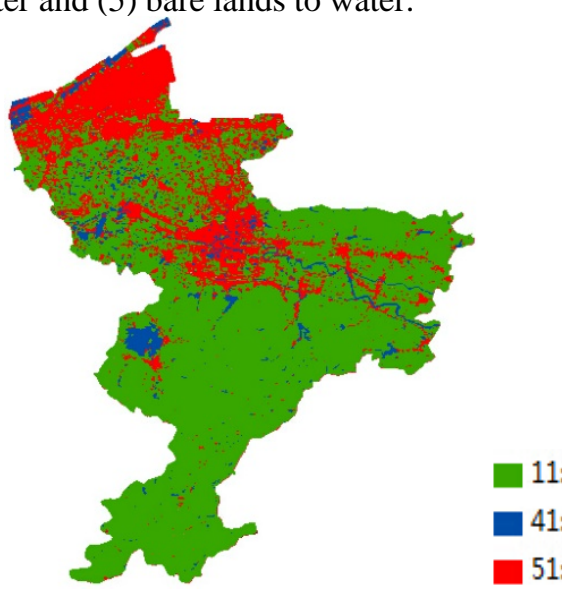

Figure 5. the classification results before Yuyao flooding disaster

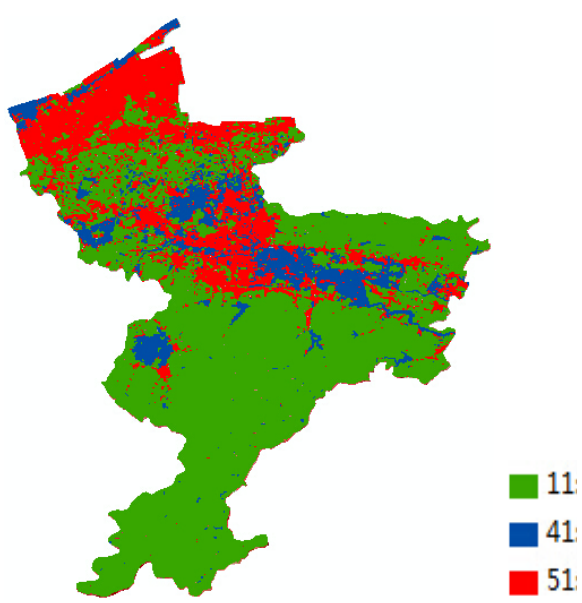

Figure 6. the classification results after Yuyao flooding disaster

Image classification results before and after floods are used in change detection. And the change detection result is shown in Fig. 7. According to the analysis of the categories changes, the flooded areas are then extracted from the change detection results. 


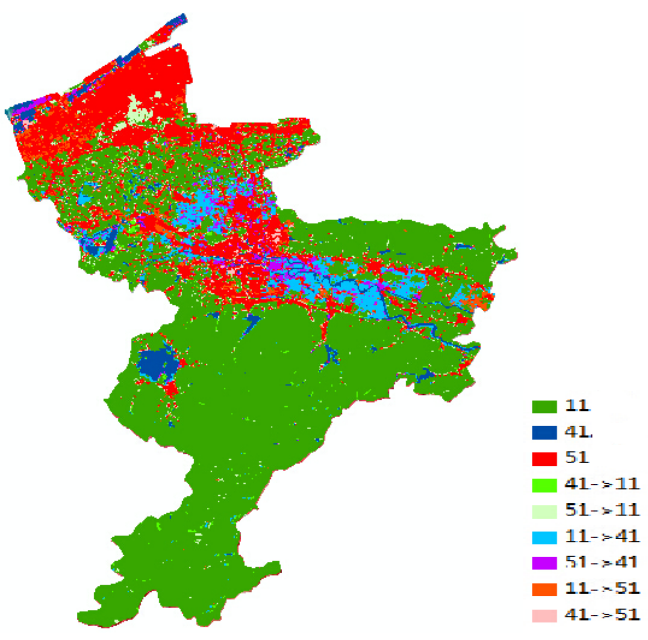

Figure 7. Image change detection results of Yuyao

\section{Accuracy and efficiency analysis}

In this experiment, we selected 7000 test samples using the stratified random sampling method. The overall classification accuracy reached $94.67 \%$ and the Kappa coefficient reached 0.907 . Particularly, for the water type, the producer's and user's accuracy are $92.79 \%$ and $92.70 \%$, respectively.

From Fig. 4, we can see that the sample library is used, which greatly reduce the workload of manual sample selection. Artificial sample selection takes roughly 20-30 minutes, but sampling from the sample library only need 2 seconds, which can meet the time requirements of affected areas extraction under a state of emergency.

\section{CONCLUSION}

In China, the research of disaster classification mainly focuses on the classification of disaster types $[6,12]$ and the classification of disaster risk management [13]. And the study of disaster reduction related applications mainly focuses on the damage assessment methods [10-11]. In this paper, an affected objects classification system for disaster mitigation with remote sensing images is proposed according to the needs of disaster reduction related applications. Further, the affected objects subdivision system for floods is established. Taking Yuyao flooding disaster as an example, rapid extraction of flooded areas is realized using automatic classification and change detection with HJ-1 satellite images. Results show that the accuracy of the extracted flooded areas and the efficiency of the rapid extraction methods can meet the needs under a state of emergency. What is more, the proposed classification system considers characteristics of disasters, information sources and disaster assessment requirements, therefore, the results supported by this system can be used in the disaster comprehensive evaluation. In this way, remote sensing based damage assessment results can be directly as an input for comprehensive evaluation models.

\section{ACKNOWLEDGMENT}

This work was supported by the Major Project (civilian part) of China High-Resolution Earth Observation (03Y30B06-9001-13/15-01) and Major Projects of China Academy of Sciences (KZZB-EW-07-02).

\section{REFERENCES}

[1] Ministry of Civil Affairs of the People's Republic of China, "National Disaster Reduction Center of China released information on national natural disasters in 2013,” Jan 2014

[2] Ministry of Civil Affairs of the People's Republic of China, "National Disaster Reduction Center of China released information on national natural disasters in 2014,” Jan 2015.

[3] Volkov Alexey, Khizhnichenko Vitaliy, "Disaster monitoring using remotely sensed data from Russian earth observation satellites," International Geoscience and Remote Sensing Symposium (IGARSS), Sydney: Institute of Electrical and Electronics Engineers Inc. Jun 1999, pp.191-193, doi: 10.1109/IGARSS.1999.773443

[4] ZHANG Jin-cun, WEI Wen-qiu, MA Wei, "A Research on the Remote Sensing Monitoring and Analysis System of Flood Disaster,” Journal of Catastrophology, Vol. 16,Jan 2001, pp. 39-44.

[5] Sun Siheng, Zheng Xinjiang, "Sandstorm Monitoring by Satellite Remote Sensing and Service for Reducing Disaster Losses," Science of Surveying and Mapping, Vol.25, Feb 2000, pp.33-37.

[6] Bo Fenxian, "Reseach on disaster classification system," Journal of Catastrophology, Vol.11, Jan 1996, pp.6-10.

[7] QIAO Cheng, LUO Jian-cheng and WU Quan-yuan, “ObjectOriented Method Based Urban Building Extraction from High Resolution Remote Sensing Image,” Geography and GeoInformation Science, Vol.24, May 2008, pp. 36-39.

[8] Yu Jie, Liu Zhenyu and Yan Jie, "Road Network Extraction of High Resolution SAR Image in Combination with Statistics and Shape Features," Geomatics Information Science of Wuhan University, Vol.38, Nov 2013, pp. 1308-1312, doi: 10.13203/j.whugis2013.11.004

[9] XIE Fei,YANG Shu-wen and LI Yi - kun, "A Method for Automatic Extraction of Debris Flow Basedon SPOT5 Image," Remote Sensing for Land\&Resouces, Vol. 3, Mar 2012, pp.78-83.

[10] TANG Ai-ping, XIE Li-li and TAO Xia-xin, "The Definition of Natural Disaster and Disaster Grade Scale,” Journal of Natural Disaster, Vol.8,Mar 1999, pp. 61-65.

[11] Ke Han, "The Research of Natural Disaster Management and Rescue System in China” Wuhan University of Science and Technology, 2007.

[12] WANG Li-li, LIN Wen, "Research and analysis of three-layer classification system of disaster events in China," Journal of Natural Disaster, Vol.20, Jun 2011, pp. 1-5, doi:10.13577/j.jnd.2011.0601

[13] LIU Xilin, SHANG Zhihai, "Establishment of integrated classification of natural disaster risk in China,” Journal of Natural Disaster, Vol.22, Jun 2013, pp. 1-7, doi:10.13577/j.jnd.2003.0601. 